2. National Drug and Alcohol Research Centre. Australian Bureau of Statistics data on opioid overdose deaths. Sydney: National Drug and Alcohol Research Centre, 2002.

3. National Drug and Alcohol Research Centre. Australian Drug Trends 2001: Findings of the Illicit Drug Reporting SystemMonograph Number 48. Sydney: National Drug and Alcohol Research Centre, 2002.

4. Weatherburn D, Jones C, Freeman K, Makkai T. Supply control and harm reduction: Lessons from the Australian heroin 'drought'. Addiction 2003, 98: 83-91.
5. Foong K and Gray J. NSW Drug Summit Update. $N S W$ Public Health Bull 2000: 11 (4): 52-53.

6. Warner-Smith M, Lynskey M, Darke S, Hall W. Heroin overdose: Prevalence, correlates, consequences and interventions-Monograph No. 46. Sydney: National Drug and Alcohol Research Centre, 2000.

7. Weatherburn D, Jones $\mathrm{C}$, Freeman $\mathrm{K}$, Makkai T. The Australian Heroin Drought and its Implications for Drug Policy-Crime and Justice Bulletin No. 59. Sydney: NSW Bureau of Crime Statistics and Research, 2001.F?

\title{
DROPS: AN AUTOMATED WEB-BASED SYSTEM FOR THE REPORTING OF DRUG RELATED HEALTH STATISTICS IN NSW
}

\author{
Marianne Jauncey \\ NSW Public Health Officer Training Program \\ NSW Department of Health
}

\section{Devon Indig}

Centre for Drug and Alcohol

NSW Department of Health

\section{Jill Kaldor}

Centre for Epidemiology and Research

NSW Department of Health

The Centre for Drug and Alcohol and the Centre for Epidemiology and Research at the NSW Department of Health have developed a web-based interface that allows access to reports on drug related morbidity and mortality in NSW. This article introduces this system, the Drugs Related Outcomes: Population Surveillance (DROPS), describing its development and how it can be used to access drug-related health statistics for the NSW population.

\section{BACKGROUND}

A range of health data related to the use of illicit and injecting drugs is routinely collected for the NSW population; however, until recently there was no system to analyse and regularly report on these data. Also, published information on this topic has tended to be presented at the state level, rather than the area health service level.

The regular reviewing and reporting of drug and alcohol data is useful in a variety of ways, to a range of key stakeholders interested in the prevention of drug-related harm and the provision of drug and alcohol services in NSW. This information can be used for: informing strategic management and policy development; monitoring trends and assisting with response planning; and providing feedback for improved service delivery.

The Centre for Drug and Alcohol and the Centre for Epidemiology and Research perceived a need for an automated system to report on routinely collected data in the drug and alcohol field, which would assist with the preparation of responses to requests for data and also provide regular reports for use in surveillance and planning. Consequently, the Centres collaborated in the development of a web-based interface that contains automaticallyupdated reports on drug related morbidity and mortality in NSW. The system is called Drug Related Outcomes: Population Surveillance (DROPS). A pilot version was tested within the Centre for Drug and Alcohol in mid-2003. After feedback and revision, DROPS was released in early 2004 to a restricted audience of nominated staff in the area health services working in the drug and alcohol field, and staff within the Centre for Drug and Alcohol.

\section{DATA USED IN DROPS}

Deaths from opiate overdose is perhaps the most widely used indicator of harm caused by illicit drugs. However, not all injected drugs are illicit and not all illicit drugs are injected. Consequently, DROPS includes data on other drugs and on certain communicable diseases that also indicate levels of harm in the community related to injected and illicit drug use.

Statistics related to psychostimulants (cocaine and amphetamines) and benzodiazepines have been included because these drugs have a high potential for harm in the injecting population. ${ }^{1-3}$ Data on notifications of hepatitis 
C are included, as it is estimated that over 90 per cent of all new infections in Australia are caused through shared injecting equipment. ${ }^{4}$

Reports are currently produced in DROPS on the following indicators within four major topic areas:

- opiates: deaths and ambulance attendance at overdose;

- psychostimulants: deaths and emergency department presentations;

- benzodiazepines: deaths and emergency department presentations;

- hepatitis $C$ : notifications of diagnosis.

For each of the above indicators the following information is provided at a state and area health service level using the most recently available data:

- yearly totals for the last five years;

- the age and sex of cases for the last complete calendar year;

- monthly totals for the most recent 24 months.

The data used in DROPS are read from a SAS-based data warehouse, ${ }^{5}$ which was built and is maintained by the Centre for Epidemiology and Research. The data warehouse is known as the Health Outcomes Information and Statistical Toolkit (HOIST), and contains a comprehensive range of datasets relevant to population health. The main sources of data currently used to compile reports in DROPS are:

- Australian Bureau of Statistics: Mortality Collection;

- Ambulance Service of NSW Case Sheet Database;

- NSW Emergency Department Data Collection;

- NSW Notifiable Diseases Database;

- Australian Bureau of Statistics population estimates.

Data sources are updated regularly on HOIST, with the frequency dependent on the source data. For example, updates for emergency department data occur each day while mortality data is updated annually. The web pages in the DROPS system are created using SAS for Windows to analyse data available in HOIST. ${ }^{5}$ The pages are updated automatically once a week, and include any new data that may have been added to HOIST during that week.

\section{HOWTO USE DROPS}

After logging onto the DROPS system, a user can 'click' their way through the various reports. Navigation is easy and intuitive. The four major topic areas are listed at the top of the page with the relevant indicator below. Once an indicator is chosen, a particular report can be chosen by another 'click', along with the choice of geographic area of interest for that report.

For example, under the topic area of opiates, a user may choose between the indicators of ambulance attendance at overdose or opiate-related deaths. Once the indicator has been chosen, the user may choose between reports of:

- yearly totals for the last five years;

- the age and sex of cases for the last calendar year;

- monthly totals for the last 24 months.

Each of these reports is available for the state and area health service populations.

There are currently over 350 reports in DROPS. Each report consists of a coloured bar graph to view any trend in the data; a table containing the numeric data that is displayed in the graph; and some standardised text that explains the source(s) of the data and provides guidance on how to interpret the data. Figures 1 and 2 show parts of two typical reports, which include a navigation section, a graph, and a data table with supporting text. Figure 1 shows opioid related deaths each year for the last five years, from the most recently available data. Figure 2 shows the number of notifications for hepatitis $\mathrm{C}$ each month for the last 24 months. Note that these figures do not show a few paragraphs of standard text associated with the report. It is likely that the data displayed for June and July 2004 in this report were not complete when this report was produced due to delays in reporting new cases.

\section{ACCESSTO DROPS}

Due to the sensitive nature of the data available in DROPS, it is only accessible on a secure server by staff of the NSW Department of Health and the area health services who have signed a confidentiality agreement. Users must abide by the NSW Health Privacy Manual (Version 1$),{ }^{6}$ and agree to use the information in DROPS for official NSW Health purposes only. Further, users agree not to pass on any information contained in DROPS to non-authorised users. Once the confidentiality agreement is signed and submitted to the Centre for Drug and Alcohol, the person is assigned a username and password to enable them to access the DROPS system. As at June 2004, there were 70 registered users with DROPS, covering all area health services.

\section{FUTURE DROPS EXPANSION}

The popularity and utility of the DROPS system has created a demand for it to expand. Examples of additional data currently under investigation for inclusion include:

\section{Specialist drug and alcohol treatment}

- NSW Minimum Data Set for Drug and Alcohol Treatment Services (for example, principal drug of concern and main service provided);

- NSW Pharmacotherapy Treatment (for example, methadone and buprenorphine clients);

- Brief Treatment Outcome Measure for Pharmacotherapy clients (for example, key outcome measures such as reduced drug use and improved social functioning). 
FIGURE 1

DRUG RELATED OUTCOMES: POPULATION SURVEILLANCE (DROPS), OPIOID-RELATED DEATHS, PERSONS OF ALL AGES, NSW, 1998-2002.
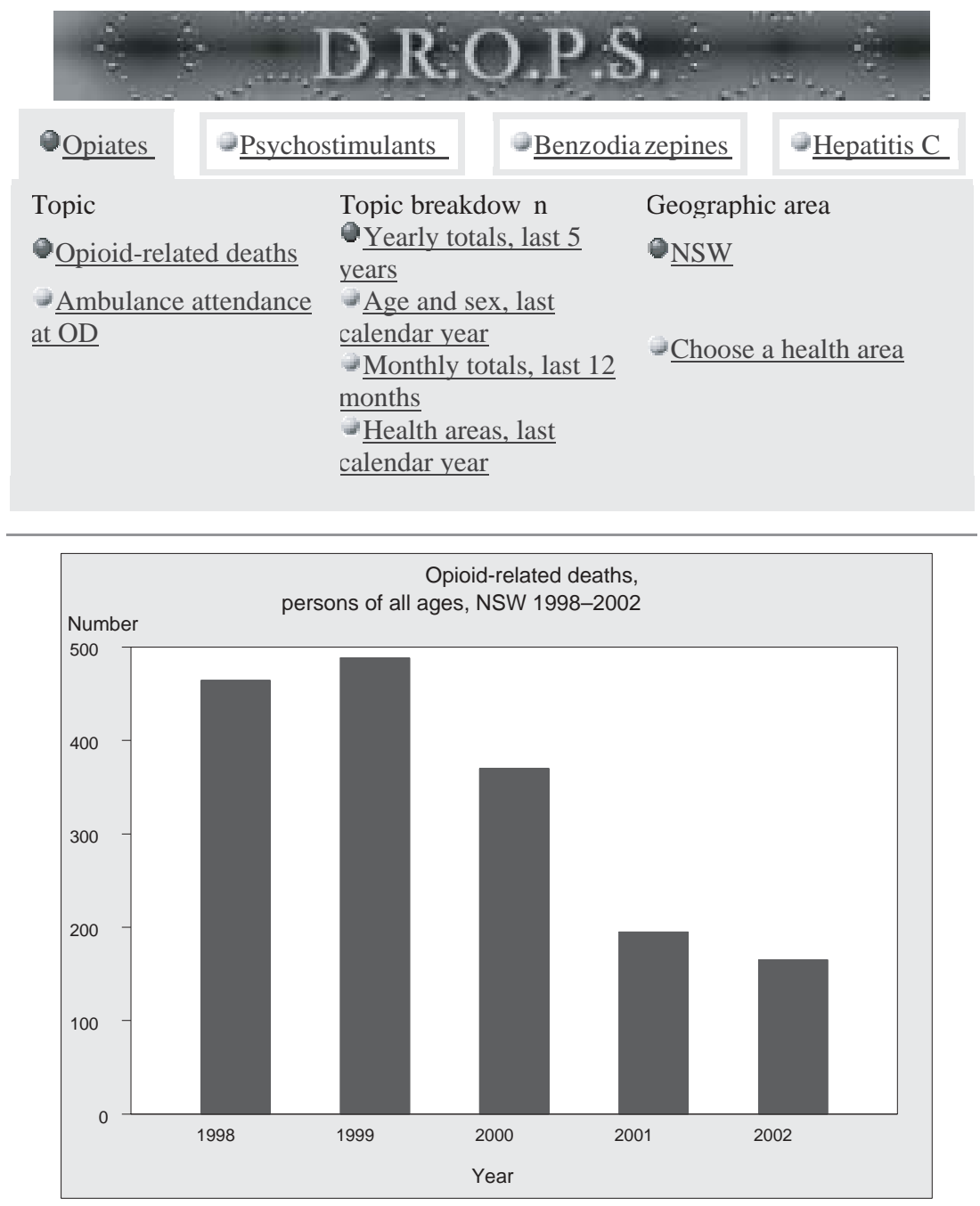

Opioid-related deaths, persons of all ages, NSW 1998-2002

\begin{tabular}{lrrrrr} 
& Year & & & & \\
& 1998 & 1999 & 2000 & 2001 & 2002 \\
\hline $\begin{array}{l}\text { Number } \\
\begin{array}{l}\text { Rate per } \\
\text { million }\end{array}\end{array}$ & 464 & 488 & 370 & 195 & 165 \\
& 73.3 & 76.3 & 57.3 & 29.9 & 25.1
\end{tabular}

\footnotetext{
Note: Data are reported by year of registration of death and place of residence at time of death. Opiate related deaths include mental and behavioural disorders due to use of opioids, accidental opioid overdose, accidental polydrug overdose with opioid toxicity, mental and behavioural disorders due to polydrug use with opioid toxicity, and mental and behavioural disorders due to polydrug use with mental and behavioural disorder due to opioids. These were classified according to the ICD-10 codes: F11; X42, X44 in association with poison codes T40.0-T40.4, T40.6; F19 in association with poison codes T40.0-T40.4, T40.6; and F19 in association with F11. Deaths due to polydrug use where toxic levels of one or more drugs were found are reported in all relevant sections of DROPS. A death due to polydrug use may therefore be reported more than once in DROPS.

Source: ABS mortality data and population estimates (HOIST). Centre for Epidemiology and Research, NSW Department of Health.
}

Source: Drug Related Outcomes: Population Surveillance (DROPS); Health Outcomes Information and Statistical Toolkit (HOIST), Centre for Epidemiology and Research, NSW Department of Health. 


\section{FIGURE 2}

DRUG RELATED OUTCOMES: POPULATION SURVEILLANCE (DROPS), HEPATITIS C NOTIFICATIONS BY MONTH, NSW, AUGUST 2002 TO JULY 2004.
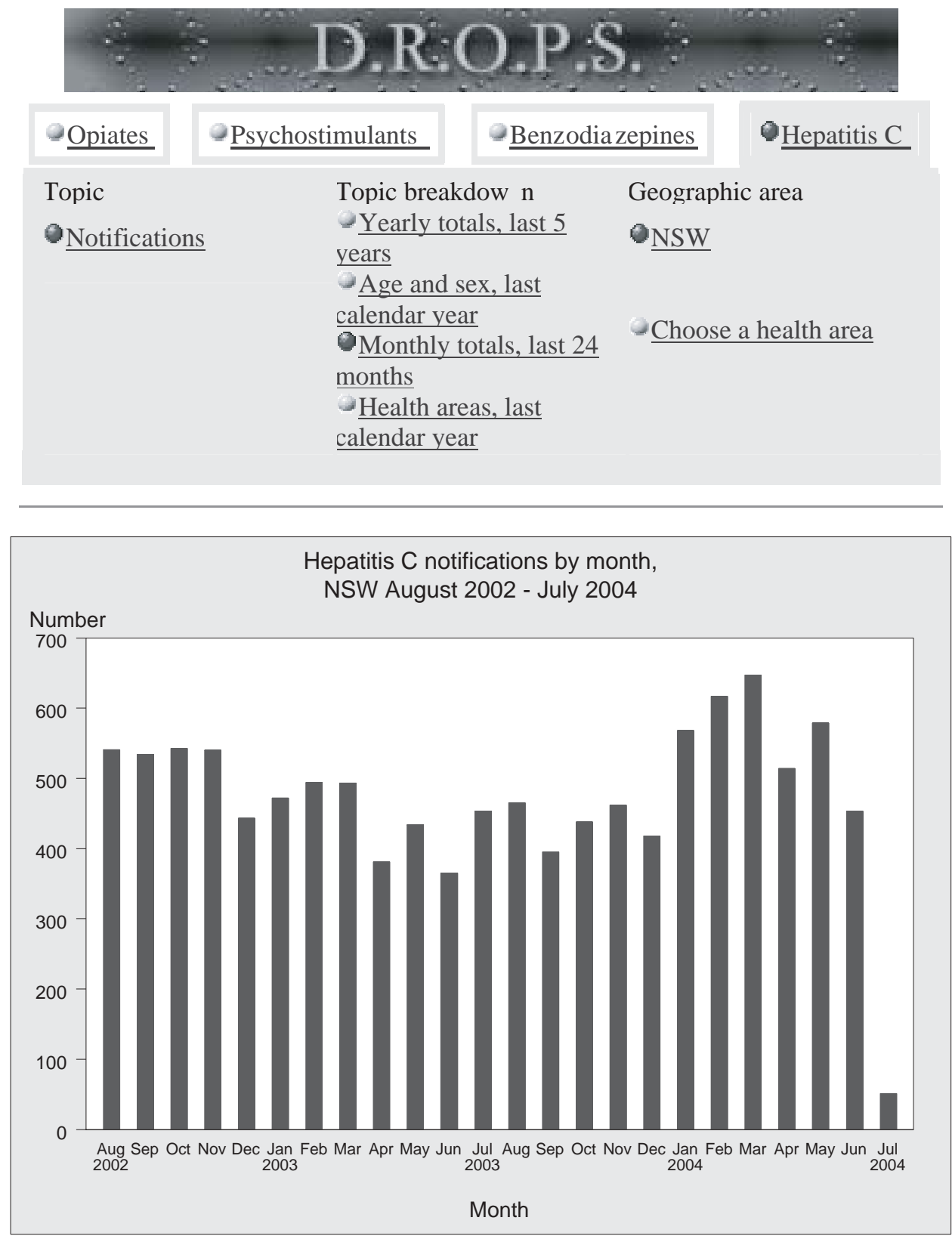

Hepatitis C notifications per month, NSW August 2002 - July 2004

$2002 \quad 2003 \quad 2003 \quad 2004 \quad 2004$

Aug Sep Oct Nov Dec Jan Feb Mar Apr May Jun Jul Aug Sep Oct Nov Dec Jan Feb Mar Apr May Jun Jul

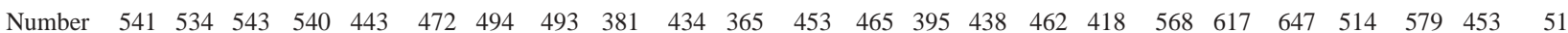

Note: Notifications received as at 16 July 2004. Lower numbers at the end of the reporting period may be due to a lag period in receiving and processing notifications.

Source: Communicable Diseases Branch NSW Notifiable Diseases Database and ABS Population Estimates (HOIST). Centre for Epidemiology and Research, NSW Department of Health.

Note: It is likely that the data displayed for June and July 2004 in this report were not complete when this report was produced due to delays in reporting new cases.

Source: Drug Related Outcomes: Population Surveillance (DROPS); Health Outcomes Information and Statistical Toolkit (HOIST), Centre for Epidemiology and Research, NSW Department of Health. 


\section{Alcohol statistics}

- alcohol-related deaths;

- alcohol-related emergency department presentations;

- alcohol-related hospital inpatient admissions;

- alcohol-related motor vehicle accidents and deaths.

\section{CONCLUSION}

The response to the development of DROPS has been positive. Timely and accessible statistics on drug-related morbidity and mortality assist the planning of service delivery to improve the health of clients. These statistics can be used to monitor trends and could offer an early warning system around emerging issues as they provide supplementary evidence to that received by clinical and frontline services. Timely and accessible data can also assist evidence-based policy development and program management, and enable the equitable allocation of resources on the basis of need.

DROPS is a major advance in improved access and availability of drug related health statistics to the drug and alcohol workforce across the state.

\section{ACKNOWLEDGEMENTS}

The authors would like to thank Dr Lee Taylor and Dr Tim Churches for their work in developing DROPS.
For more information about DROPS contact Devon Indig at the Centre for Drug and Alcohol, NSW Department of Health, by phone on (02) 93919220 or by email on dindi@doh.health.nsw.gov.au.

\section{REFERENCES}

1. Hando J, Topp L, Hall W. Amphetamine-related harms and treatment preferences of regular amphetamine users in Sydney, Australia. Drug Alcohol Depend 1997; 46(1-2): 105-113.

2. Fry CL, Bruno RB. Recent trends in benzodiazepine use by injecting drug users in Victoria and Tasmania. Drug Alcohol Rev 2002; 21(4): 363-367.

3. Darke S, Topp L, Ross J. The injection of methadone and benzodiazepines among Sydney injecting drug users 19962000: 5-year monitoring of trends from the Illicit Drug Reporting System. Drug Alcohol Rev 2002; 21(1): 27-32.

4. Law MG, Dore GJ, Bath N, Thompson S, Crofts N, Dolan K, Giles W, Gow P, Kaldor J, Loveday S, Powell E, Spencer $\mathrm{J}$, Wodak A. Modelling hepatitis $\mathrm{C}$ virus incidence, prevalence and long-term sequelae in Australia, 2001. Int J Epidemiol 2003; 32: 717-724.

5. SAS Institute. The SAS System for Windows Version 8.02. Cary, NC: SAS Institute, 2001.

6. NSW Health Privacy Manual (Version 1). Sydney: NSW Department of Health, 2004. Available online at

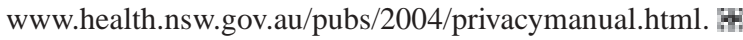

